\title{
Concept of an Experimental Setup for Testing the Technology for the Formation of New Anti-Corrosion Coating Materials Using Low-Temperature Supersonic Heterogeneous Flows
}

\author{
Sergey A. Borisov, Yulia I. Glukhovskaya, Sergey V. Dobrovolskiy, \\ Petr V. Nikitin, Igor V. Podporin
}

Moscow Aviation Institute (MAI), Volokolamskoe highway, Moscow, Russia

\begin{abstract}
The article describes the creation of the concept of an experimental setup for testing the technology for the formation of new anti-corrosion coating materials using low-temperature supersonic heterogeneous flows, which allows scientific research to be carried out in a wide range of tasks of practical interest in order to create protective coatings for metal products operating under conditions of erosion and corrosion wear.
\end{abstract}

Keywords - anti-corrosion coatings, heterogeneous flow, low-temperature gas-dynamic technology, supersonic gas-dynamic accelerator.

\section{Introduction}

Currently, the world is searching for highperformance technologies that protect metal structures susceptible to erosion and corrosion wear. It is necessary to develop a facility on which experiments could be conducted to solve these problems that verify theoretical developments in this field.

DOI: $10.18421 /$ TEM92-19

https://doi.org/10.18421/TEM92-19

Corresponding author: Sergey V. Dobrovolskiy, Moscow Aviation Institute (MAl), Moscow, Russia.

Email: dobrovolskiy_s_v@mail.ru

Received: 20 February 2020.

Revised: 02 April 2020.

Accepted: 10 April 2020.

Published: 27 May 2020.

(c) BY-NC-ND (C) 2020 Sergey V. Dobrovolskiy at al; published by UIKTEN. This work is licensed under the Creative Commons Attribution-NonCommercial-NoDerivs 3.0 License.

The article is published with Open Access at www.temjournal.com
The study of the use of low-temperature supersonic heterogeneous flows to obtain new coating materials on the surfaces of objects operated under the influence of erosion and corrosion factors is a priority task, in which a huge economic potential is hidden. The result of the study will be the creation of a technological process for the formation of new coating materials for protecting pipelines from erosion and corrosion factors in an experimental setup designed to test this technology.

Thus, the creation of an experimental setup is an important scientific and practical task, the first step of which is the development of the concept of an experimental setup for testing the technology for the formation of new anti-corrosion coating materials using low-temperature supersonic heterogeneous flows.

\section{Theoretical Basis}

The concept of the experimental setup is based on the formation of a high-speed heterogeneous flow and its interaction with a solid surface. When solid particles from a heterogeneous stream hit the surface, most of the kinetic energy of the particles moving in the stream passes into heat, causing a significant increase in local temperature and deformation of particles in the contact zone, the energy of which, when interacting with the surface, goes into different forms of energy, including the energy of formation of interatomic bonds.

When conducting research, it was found that the process of particle interaction is similar to explosion welding. In the impact zone, mutual shear deformation of the crystal lattices in the materials of the particles and the substrate, activation of the conjugated surfaces (including cleaning of oxide films) occurs. In addition, dynamic effects arise in the contact patch (for example, dynamic diffusion) as 
a result of a local pressure pulse and an increase in temperature at the moment the particle hits the substrate. This whole complex of processes provides a high level of adhesive and cohesive properties of products, as well as their strain hardening throughout the thickness.

The mass fraction of particles that form the product in a heterogeneous flow does not exceed $10 \%$ of the mass fraction of the carrier gas [1].

The gas-dynamic method allows obtaining both uniform composition and changing coating properties, obtaining composite metal products. To obtain the desired properties and composition of materials, the calculated amount of particles with sizes from 5 to $25 \mu \mathrm{m}$ is fed into the stream. The required composition of heterogeneous powders in the calculated proportion is prepared in a special gasdynamic mixer. The composition with the necessary parameters (pressure and temperature) enters the prechamber and then accelerates in the supersonic nozzle to the speed calculated for the given composition. The pressure and temperature of the carrier gas are chosen so that the speed of the particles at the outlet of the acceleration section corresponds to the calculated one, and their temperature does not exceed $20 \ldots 50 \%$ of the melting temperature of the particle material. The main condition is that the product is formed due to the high level of kinetic energy of the particles, while the temperature (enthalpy) of the flow is much lower than their melting temperature.

The scientific concept of coating formation is based on the physical principle of lowering the level of static temperature of the gas phase, and at the same time particles, with a significant increase in the speed of the heterogeneous flow in the accelerating section.

As a result of the implementation of shock wave loads, as well as the dissipation of kinetic energy, physicochemical and phase transformations occur in the collision spot. All these processes accompany both the change in the crystal lattice of the starting materials and the possible formation of materials with new properties.

Thus, the detected signs make it possible to assert that using gas-dynamic methods, it is possible to create various coatings, set their properties, and control the process with high accuracy.

\section{Methodology}

The development of the concept of an experimental setup (ES) was carried out with the aim of creating a laboratory base for a wide range of experiments on the formation of various coatings on samples of flat and cylindrical shapes using the lowtemperature gas-dynamic method (LTGDM) [2]. The developed experimental setup and the composition of studies conducted at ES should ensure the creation of a technology for applying various coatings, which, in turn, will form the scientific basis for the creation of industrial plants and industrial cycles for the formation of protective coatings by the LTGDM [3].

The physical principle of coating with LTGDM technology is implemented using the developed block diagram of the method implementation presented in the Figure 1. compressed air from the compressor station is pumped into the cylinder compressed air enters through pneumatic lines, which are equipped with shut-off valves and pneumatic regulators, to gas flow control systems 3 and 3', mounted on a common console of a gasdynamic setup. Part of the compressed air enters the powder dispenser control system 3 , in which a gaspowder suspension is created with the necessary mass flow rate of the powder and then transported to the mixing chamber with nozzle block 2 . The main part of the compressed air with the parameters specified by the regulator $3^{\prime}$ is supplied to the carrier gas heater 4 for heating to the design temperature. The heated gas enters the mixing chamber 2 and subsequently acts as the carrier gas of the powder.

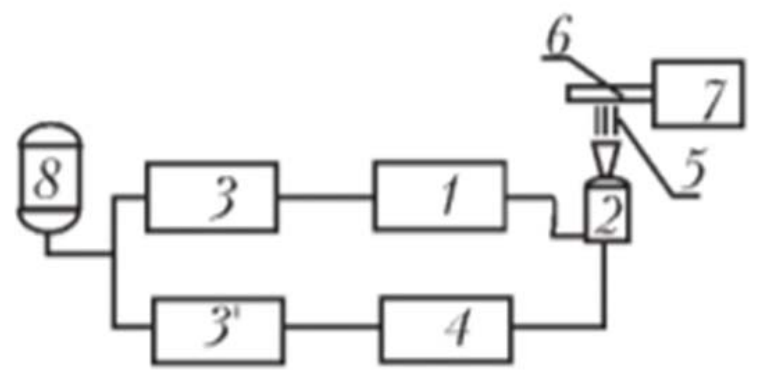

1 - powder dosing and feeding system, 2 - mixing chamber with nozzle block, 3 - control system for parameters and mass flow of carrier gas, 3' - powder dispenser control system, 4 - carrier gas heater, 5 - heterogeneous supersonic flow, 6 - workpiece, 7 - workpiece movement mechanism, 8 - compressed air cylinder

Figure 1. Setup block diagram for implementing LTGDM-technology 
The optimal ratio of the mass flow rate of the carrier gas and powder are set by systems 3 and 3', gas-powder suspension is gas-dynamically mixed in the mixing chamber 2, in which the calculated parameters pressure and temperature of the heterogeneous mixture are provided. Next, the heterogeneous mixture enters the accelerating nozzle block (accelerator), forming a supersonic heterogeneous flow 5. The jet is directed to the workpiece (substrate) 6, fixed in the workpiece movement mechanism 7 , providing its necessary rotational and reciprocating motion.

The coating mode for this pair of "powdersubstrate" is determined by the speed of the powder particles at the moment of their collision with the base and speed of movement. The speed of the powder particles depends on the temperature and pressure of the carrier gas in the mixing chamber. The set of values of all these parameters determines the technological mode of coating.

\section{Definition of the Main Technological Characteristics}

Before proceeding with the design of the ES gasdynamic path, it is necessary to analyze the entire technological cycle that remains to be completed. For example, the designed ES is designed to form a protective coating on the inner surface of pipes of a particular assortment. To conduct experiments to determine the resistance of the coating to erosion and corrosion, flat samples of certain sizes will be required. In addition, the study of the adhesion and cohesion properties of coatings will require the formation of coatings on the outer surfaces of a cylindrical shape [4].

To complete the tasks, we need to determine the main parameters of the designed stand.

\section{Polydispersity of Powders}

To form high-quality coatings using the lowtemperature gas-dynamic method, it is necessary to use polydisperse powders with micron range particle diameters. In this case, the polydispersity of the powders (range of particle diameters) is determined by the density of the material of the initial powder. For example, for heavy metals (tungsten, tantalum, etc.), polydispersity of the powders can vary from 1 $\mu \mathrm{m}$ to $5 \mu \mathrm{m}$. For metals of medium density (steel, copper, zinc, etc.), polydispersity is $3-15 \mu \mathrm{m}$. For light metals (aluminum, titanium, etc.), it varies in the range of 3-20 $\mu \mathrm{m}$ (permissible up to $50 \mu \mathrm{m}$ ).

The indicated ranges of the polydispersity of the powders are due to the fact that the heavier the particle, the more difficult it is to accelerate it to the speed at which the coating is formed. This speed is called critical one. It is a function of many parameters and determines the efficiency of the process and high quality of coatings [5].

\section{Thickness and Shape of Coatings}

The study of the properties of protective coating samples from various materials obtained using the low-temperature gas-dynamic method has established that the high quality of coatings allows the use of coatings with a thickness not exceeding $25 \mu \mathrm{m}$ to protect steel surfaces of metal structures from atmospheric corrosion, impacts and other factors. Nevertheless, now it may be necessary to form coatings with a thickness of more than $150 \mu \mathrm{m}$ and the designed setup should provide similar coating thicknesses [6].

In engineering, metal structures of various shapes are used; however, designs of a flat or axisymmetric shape (pipe, sheet, corner, tee, channel, etc.) are prevailing.

\section{Ensuring the Necessary Duration of the Experiments}

The main parameter limiting the duration of the experiments is the stock of compressed air located in the gas cylinder receiver station. The air volume should be sufficient to maintain a constant working pressure $(5.0 \mathrm{MPa})$ in the pneumatic system of the gas-dynamic stand during the setup time of the equipment to reach the operating parameters and during the formation of coatings on experimental samples [7].

Since the program of planned experiments involves the study of coating formation processes for a number of powders of various fractions and densities, and in addition, it will be necessary to study the operating modes of the setup from the point of view of preparing data for industrial production, it is necessary to have a significant supply of air for carrying out adjustment and tuning activities [8].

\section{Experimental Setup}

The design of a high-performance experimental setup for the formation of protective coatings on substrates of various configurations was carried out.

The main element of the gas-dynamic stand is the path, in which at the final stage a two-phase supersonic flow is formed using special accelerators (nozzles). Depending on the technological problems being solved at the production, various devices can be used as accelerating channels. Their profiles of these accelerating devices are calculated using the basic principles of thermogasdynamics and heat transfer theory as applied to heterogeneous media. 
Moreover, the production of profiled channels is carried out according to the class of high surface purity [9].

The developed concept of the stand should allow full automation of the process. It determines science intensity of the equipment designed for the production.
The indicated technical conditions can be implemented on a gas-dynamic bench based on this concept and the above analysis, circuit diagram of which is shown in the Figure 2.

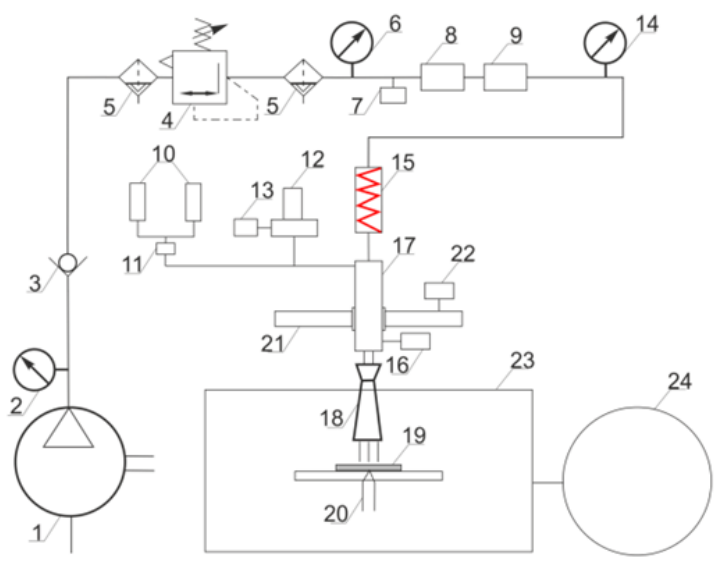

1. Compressor

2. Compressor pressure gauge

3. Shut-off valve

4. Air preparation module 1

5. Filter regulator

6. Pressure gauge in the line

7. Air and solid phase control unit

8. Electropneumatic valve

9. Air flow sensor

10. Ejector powder dispensers

11. Dispenser shut-off valve

12. Powder rotary dispenser

13. Control unit of the rotary dispenser

14. Air pressure gauge at the inlet to the mixer
15. Ohmic air heater

16. Control unit for the temperature of the air at the entrance to the accelerator

17. Mixer

18. Heterogeneous flow accelerator

19. Formed coating

20. Thermocouple on the test sample

21. Scanning device, robot

22. Control unit of the scanning device

23. Working chamber

24. Setup for collecting unused particles, air purification

25. Pipeline

26. Control cable

Figure 2. Schematic diagram of the setup

The main setup systems are:

- Main pneumatic system;

- Dispenser system;

- Heater system;

- Feed (movement) system;

- Ventilation system of the booth;

- Diagnostic and control system;

- Sound insulation system.

\section{Main Pneumatic System}

For the formation of a two-phase mixture in the desired ratio of mass concentrations of particles and carrier gas (air), as well as the delivery of this mixture to the accelerator chamber (supersonic nozzle), two pneumatic systems are provided in the booth scheme (Figure 2.): pneumatic system for supplying the main flow of carrier gas and pneumatic system for powder dispenser. Both pneumatic systems are connected through a manifold and gearbox to the compressed air supply system. The pressure level at the inlet and in each of the pneumatic systems is controlled by manometers and pressure sensors [10].

To implement the working process of coating, highpressure pneumatic system must provide the necessary (calculated) gas flow rate at the appropriate pressure. The pneumatic system consists of a reduction gear, pressure of which is controlled by a pressure gauge, check valve, gas heater, mixing chamber for the phases of the carrier gas (air) and powder, with predetermined costs. Pressure gauges, temperature sensors and thermocouple in the nozzle control the parameters of air and heterogeneous mixture [11].

The pneumatic system works are the following: carrier gas reduced to the required pressure by the 
pressure reducer enters the ohmic heater through the check valve, in which the carrier gas is heated to the required (calculated) temperature. The electrical parameters of the heater are controlled by a voltmeter, ammeter and other control sensors with access to the recorder [12]. After the heater, air stream enters through the high-pressure flexible hoses into the mixing chamber, where the main stream is mixed with the heterogeneous stream from the ejector-type powder batcher. The ejectordispenser must provide a smooth adjustment of the mass flow rate of the powder during the coating process and is connected to the mixing chamber by flexible high-pressure hoses, which provide the possibility of moving the mixing chamber with the nozzle [13].

After the mixing chamber, heterogeneous stream enters the supersonic accelerator, where it accelerates to the required speeds. The type of accelerator is determined by the shape of the substrate on which the coating is formed.

\section{Heater System}

The heater system consists of an ohmic heater and device that controls input electrical power.

The ohmic heater consists of several seriesconnected stainless steel pipes of a certain length. The pipes are curled in spirals to save space. An adjustable electric power is supplied to each pipe, causing its walls to heat up. Thus, the air flow passing inside the pipes is heated to the required temperature.

Heater tubes are connected to thyristor voltage converters.

To achieve the maximum possible electric power, it is planned to use two converters connected in parallel. Each converter is equipped with a remote control connected to the recorder. Each converter is connected to water supply and sewage systems to ensure removal of excess heat [14].

\section{Feed (movement) System}

Feed (movement) system changes the position of the mixing chamber and supersonic nozzle according to a given algorithm using numerical control system.

For research, three-coordinate feed system with computer numerical control (CNC) with one main direction of movement and two auxiliary ones is required. The feed in the main direction should allow the nozzle and mixing chamber assembly to move with a total weight of up to $10 \mathrm{~kg}$ with speeds reaching $1000 \mathrm{~mm} / \mathrm{s}$. These speeds are due to the requirements of calculations for the formation of thin coatings up to $25 \mu \mathrm{m}$ thick with high isotropy or for the formation of composite coatings. For auxiliary directions the displacement velocity factor is not so significant, since these types of supply are supposed to be used when centering relative to the axes.

Now it is planned to use elements of CNC machine tools or a set of linear actuators prepared for $\mathrm{CNC}$ and placed on a makeshift rigid frame as the basis of the feed system.

In the future, it is planned to use robotic manipulators to form various coatings on curved surfaces.

\section{Stand Ventilation System}

The ventilation system should ensure the sewage of excess air in the room, formed when the air flows from the nozzle, as well as the separation and filtration of powder particles that enter the atmosphere of the room during the experiments.

The system consists of exhaust hood (cabinet), highways, cyclone filter, microfilter, and exhaust fan. The air with the powder particles after passing the placement of the sample is collected in an exhaust hood or cabinet, depending on the type of sample and the nozzle used. From an umbrella or cabinet, contaminated air flows through flexible and rigid lines to a cyclone, where the particles of powder are separated from the air stream and particles are collected in a container. After the cyclone, the air flow is further cleaned in the microfilter and thrown out through the silencer into the atmosphere using a fan. System productivity is $3500-4000 \mathrm{~m} 3 /$ hour. Excessive pressure created by the fan: up to $600 \mathrm{~Pa}$.

\section{Diagnostic and Control System}

Diagnostic and control system is designed to collect and display parameters characterizing the ongoing experiments and control these parameters. This system consists of a diagnostic and control panel, rack of pressure regulators of various sensors, and parameter control devices located on the setup. In addition, when debugging a bench for using various powder materials and mixtures, laser Doppler anemometer can be used to measure the true velocity of powder particles at the exit of the nozzle [15], [16].

In addition, when debugging a bench for using various powder materials and mixtures, laser Doppler anemometer can be used to measure true velocity of powder particles at the exit of the nozzle.

\section{Sound Insulation System}

When the supersonic flow from the nozzle expires, sound pressure arises in the entire acoustic frequency range. To reduce sound pressure, it is planned to use soundproof materials on the ducts of the exhaust system, as well as sound-absorbing mats and screens 
around noise sources. Placements and screen parameters will be determined empirically using special equipment.

\section{Carrier Gas State Parameters in the Accelerator Prechamber}

Carrier gas state parameters in the accelerator prechamber of the accelerator are determined by the type of coating and its main characteristics such as adhesion, cohesion, permeability, density, etc., as well as the dimensions of the coating: thickness and characteristic size (for example, width).

All coating properties are determined by the speed and temperature of the powder particles at the time of impact on the surface to be treated.

The indicated characteristics depend on the length of the accelerator and velocity of the carrier gas at its cut.

The calculation of the geometric dimensions of the nozzle is one of the most important design procedures for the gas-dynamic path of the setup.

It was experimentally established that for the formation of high-quality coatings from corrosionresistant steel, the parameters of the state of carrier gas at the inlet of the accelerator should be static pressure $4 \mathrm{MPa}$ and static temperature of $700 \mathrm{~K}$. These parameters are achieved by regulating the pressure, as well as by regulating the electrical power supplied to the heater.

\section{Mass Flow Rate of the Carrier Gas in the Gas Dynamic Path}

The mass second flow rate of the carrier gas in the gas-dynamic path is determined by the second flow rate of the carrier gas in the critical section of the accelerator.

In the study of heterogeneous flows, it was found that if the mass concentration of the solid phase is not more than $10 \%$, then the flows of the heterogeneous mixture in the channels obey the laws of classical gas dynamics. Thus, in our case, in order to fulfill the indicated condition, the mass second flow rate of the carrier gas should at least 10 times exceed the mass flow rate of the solid phase. In addition, the mass second flow rate of the carrier gas depends on the technological operation performed on the gasdynamic setup.

\section{Providing the Necessary Composition and Velocity of the Powder Particles}

As the materials that form the coatings, it is supposed to study a whole range of powder compositions, mixtures, and compositions with a fairly wide range of properties and characteristics.
The upper limit of the particle velocity is characterized by the strength properties of the powder material and gas-dynamic parameters of the air flow in the gap between the accelerator and the substrate. In turn, the kinematics of particles is determined by their mass and flow rate. Since a considerable part of the powders and their compositions under consideration have a high density of the starting material, significant values of the velocity head of the air flow will be required to form a durable coating from them.

The velocity head at the cut of the accelerator depends on the following factors:

- air pressure difference between the entrance to the accelerator and the cutoff of it;

- accelerator inlet air temperature;

- accelerator geometry;

- geometric dimensions of the gas-dynamic path are determined based on the corresponding provisions of it.

\section{Results and Discussion}

The concept of an experimental setup has been developed to test the technology for the formation of new anti-corrosion coating materials using lowtemperature supersonic heterogeneous flows, the use of which makes possible to make research and industrial setup for creating protective coatings using a high-performance method.

The concept of an experimental setup for testing the technology for the formation of new anti-corrosion coating materials using low-temperature supersonic heterogeneous flows was created using the laws of thermodynamics and gas-dynamic calculations.

The tests performed show the correspondence of the obtained results to theoretical calculations.

\section{Conclusions}

The proposed concept of an experimental setup for testing the technology for the formation of new anticorrosion coating materials using low-temperature supersonic heterogeneous flows allows scientific research to be carried out in a lot of actual tasks in order to create protective coatings for metal products operating under conditions of erosion and corrosion wear.

The implementation of this concept of the experimental setup allows the following:

- conducting a comparative analysis of gasdynamic methods in order to identify positive and negative factors;

- working out diagnostic methods for parameters of high-speed homogeneous and heterogeneous flows; 
- analyzing the flow of a supersonic heterogeneous one when it flows onto an obstacle;

- studying the mechanism of interaction of a supersonic heterogeneous flow with a surface;

- determining the effect of heterogeneous flow parameters on the efficiency of coating formation;

- creating an algorithm for calculating the flow of a heterogeneous mixture in the elements of the gas-dynamic path for industrial design equipment and coating formation technologies.

\section{Acknowledgements}

This work was financially supported by the Ministry of Science and Higher Education of the Russian Federation, agreement № 05.607.21.0299 of December 3, 2019, unique project identification number: RFMEFI60719X0299.

\section{References}

[1].Borisov, S. A., Dobrovolskiy, S. V., Gloukhovskaya, Yu. I., \& Nikitin, P. V. (2019). Study of the Erosive Wear of Coatings by Means of a Mobile Heterogeneous Jet Flow of Limited Area. Journal of Surface Investigation: X-ray, Synchrotron and Neutron Techniques, 13(2), 261-266.

[2].Nikitin, P. V., Borisov, S. A., Dobrovolskiy, S. V., \& Glukhovskaya, Yu. I. (2016). Mathematical model of the process of leakage of a supersonic heterogeneous flow onto a flat obstacle. Surface. X-ray, synchrotron and neutron studies, 10, 1-6.

[3].Assadi, H., Kreye, H., Gartner, F., \& Klassen, T. (2016). Cold spraying - A materials perspective. Acta Materialia, 116, 382-407.

[4].Moridi, A., Hassani-Gangaraj, S. M., Guagliano, M., \& Dao, M. (2014). Cold spray coating: review of material systems and future perspectives. Surface Engineering, 30(6), 369-395.

[5].Shorinov, A. V., Volkov, A. O., \& Markovich S. E (2018). Cold gas-dynamic deposition of metal coatings on composite materials and plastics: a literature review. Bulletin of Kharkiv National Automobile and Highway University, 81.
[6].Grigoriev, S., Okunkova, A., Sova, A., Bertrand, P., \& Smurov, I. (2015). Cold spraying: From process fundamentals towards advanced applications. Surface and coatings Technology, 268, 77-84.

[7].Sun, W., Tan, A. W. Y., Marinescu, I., Toh, W. Q., \& Liu, E. (2017). Adhesion, tribological and corrosion properties of cold-sprayed CoCrMo and Ti6Al4V coatings on 6061-T651 Al alloy. Surface and Coatings Technology, 326, 291-298.

[8].Chang, Y., Mohanty, P., Karmarkar, N., Khan, M. T., Wang, Y., \& Wang, J. (2020). Microstructure and properties of $\mathrm{Cu}-\mathrm{Cr}$ coatings deposited by cold spraying. Vacuum, 171, 109032.

[9].Li, Y. J., Luo, X. T., \& Li, C. J. (2017). Dependency of deposition behavior, microstructure and properties of cold sprayed $\mathrm{Cu}$ on morphology and porosity of the powder. Surface and Coatings Technology, 328, 304312.

[10]. Xia, M., Huang, P., Cu, R. K., \& Ge, C. C. (2016). Cold sprayed W/Ni/Fe alloy coating: Microstructure and mechanical properties. Surface and Coatings Technology, 291, 376-381.

[11]. Malachowska, A. (2015). Analysis of the cold gas spraying process and determination of selected properties of metallic coatings on polymers. $\mathrm{PhD}$ Thesis. Wroclaw: University of Technology (Poland).

[12]. Metechko, L. B., Sorokin, A. E. \& Novikov, S. V. (2019). Aerospace monitoring in solving problems of modern precision agriculture. Amazonia Investiga, 8(19), 638-646.

[13]. Khayrnasov, K. Z. (2019). Modeling and thermal analysis of heat sink layers of multilayer board. Amazonia Investiga, 8(23), 664-670.

[14]. Jakupi, P., Keech, P. G., Barker, I., Ramamurthy, S., Jacklin, R. L., Shoesmith, D. W., \& Moser, D. E. (2015). Characterization of commercially cold sprayed copper coatings and determination of the effects of impacting copper powder velocities. Journal of Nuclear Materials, 466, 1-11.

[15]. Kanashchenkov, A. I., Matveev, A. M., \& Novikov, S. V. (2018). Measuring System for Use in RadarSystem Development. Russian Engineering Research, 38(11), 896-900.

[16]. Belyavskii, A. E., Novikov, S. V., Sorokin, A. E., \& Shangin, I. A. (2019). Thermal Stores in Spacecraft Heating Systems. Russian Engineering Research, 39(6), 507-509. 